Tome 40, no 24. - Juillet 1933.

Prof. Dr E. HANDSCHIN

VOYAGE D'ÉTUDES AUX ILES DE LA SONDE

et en australie Septentrionale, 1930-32

\title{
Note sur quelques Crustacés décapodes d'eau douce provenant de l'Australie septentrionale
}

par

\section{Jean ROUX}

Musée d'Histoire naturelle, Bâle.

Pendant son séjour dans le Territoire Nord du Commonwealth australien, M. le Prof. Ed. Handschin a recueilli quelques Crustacés décapodes d'eau douce qu'il a bien voulu offrir au Musée de Bâle.

Quoiqu'il ne s'agisse que d'un petit nombre d'espèces, il nous a semblé utile d'en faire un relevé, étant donné que cette région a été encore peu explorée et que, d'autre part, il se trouve parmi les formes récoltées, une espèce nouvelle pour la science.

La collection contient:

3 espèces de Palémonides:

Palaemon (Eup.) australis Ortm.

Palaemon (Eup.) carcinus rosenbergi v. Mart.

Palaemon (Macrobr.) handschini n. sp.

1 espèce de Parastacide:

Cheraps quadricarinatus v. Mart.

1 espèce de Potamonide:

Paratelphusa (Liot.) transversa (v. Mart.)

Nous donnons ci-dessous quelques détails relatifs à ces différentes espèces, nous arrêtant tout spécialement à la description de l'espèce nouvelle.

Rev. Suisse de Zool., T. ‘0, 1933. 


\section{Palaemon australis Ortmann.}

Katherine Riv. N. Australie, 4 juv.

Ces individus, quoique jeunes, répondent assez bien à la description de cette espèce donnée par DE Man et Ortmann.

Le plus grand des exemplaires n'a que $42 \mathrm{~mm}$ de longueur totale. Le rostre est dirigé horizontalement en avant et atteint l'extrémité du scaphocérite. Nous avons relevé les formules suivantes pour les dents rostrales $\frac{(2) 8}{2}, \frac{(2) 9}{3}, \frac{(2) 10}{3}, \frac{2(10)}{4}$. Il n'y a donc que 2 dents situées sur le céphalothorax au lieu de 3, comme l'indiquent les auteurs.

Les pattes I dépassent en avant l'écaille antennaire de la longueur des doigts de la pince. La longueur de cette dernière est contenue $12 / 3$ fois dans celle de l'article précédent.

Aux pattes II le carpe, plus long que le mérus, est un peu plus long que la paume de la pince et les doigts de celle-ci sont plus courts que la partie palmaire. On aperçoit 2 petites dents, dans la partie basale des doigts, mais il n'y a pas encore le feutrage qu'on remarque chez les individus adultes. La paume est légèrement aplatie, ainsi que l'indique DE MAN. Tous les articles des chélipèdes sont recouverts de fines aspérités et ornés en outre de quelques poils fins, isolés. Les pattes ambulatoires sont longues et fines.

\section{Palaemon (Eupalaemon) carcinus rosenbergi de Man.}

Katherine Riv. N. Australie, 1 ๙ิ, 2 ๙ิ jus.

Le plus grand des individus a $120 \mathrm{~mm}$ de longueur totale.

Le rostre a la forme typique pour la sous-espèce; il est relevé dans la région sus-orbitaire. Sa formule dentaire est $\frac{(3) 7+1+1}{7}$; il atteint en avant l'extrémité du scaphocérite. Chez les jeunes, le rostre dépasse de beaucoup l'écaille antennaire et se relève distinctement vers le haut; il porte des dents échelonnées sur toute sa longueur. 
Les rapports entre les divers articles des chélipèdes II correspondent à ceux que nous avons indiqués pour cette espéce. ${ }^{1}$

Voici les dimensions des pattes II, qui sont inégales.

\begin{tabular}{|c|c|c|c|}
\hline & & Gauche & Droite \\
\hline Mérus & . & $17^{\mathrm{mm}}$ & $20^{\mathrm{mm} .}$ \\
\hline Carpe . & 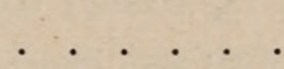 & $19 »$ & $25 »$ \\
\hline Pince. & . . . . & $33 »$ & $44 ”$ \\
\hline Paume &... & $19 »$ & $25 »$ \\
\hline Doigt & . & $14 »$ & $19 »$ \\
\hline
\end{tabular}

Ici le carpe a la même longueur que la palma; mais il peut y avoir un certain écart dans un sens ou dans l'autre. Les doigts sont toujours plus courts que la portion palmaire.

\section{Palaemon (Macrobrachium) handschini n. sp.}

Katherine Riv. N. Australie, 6 Ex. (5 $\sigma^{\wedge}, 1$ juv.)

Cette espèce est de petite taille et se rapproche des Pal. bariensis de Man, habitant Flores et de Pal. callirhoë de Man, trouvé à Bornéo. Elle en diffère cependant par plusieurs caractères.

Le plus grand individu mesure $37 \mathrm{~mm}$ de longueur totale. La carapace est recouverte, surtout en avant, de petites aspérités. Le rostre, qui commence en deçà du milieu du céphalothorax est dirigé horizontalement en avant ou légèrement vers le bas; il est droit ou bien peut présenter, au-dessus de l'œil, une légère convexité. Vu de côté, il ne parait pas étroit, car sa hauteur est contenue $3 \frac{1}{2}$ fois dans sa longueur. Sa forme rappelle celle du Pal. pilimanus de Man. Il s'étend en avant aussi loin que le pédoncule antennulaire ou un peu au-delà.

Les formules dentaires notées sont les suivantes: $\frac{(3) 10}{3} 1$ fois, $\frac{\text { (3) } 11}{3} 2$ fois, $\frac{\text { (4) } 11}{3} 4$ fois. Des dents fixées sur le céphalothorax, les deux premières sont un peu plus basses que les suivantes. L'épine antennaire est bien développée; en arrière d'elle, mais

${ }^{1}$ In: Nova Guinea, vol. XIII, 1921, p. 589, et vol. XV, 1927, p. 321. 
parfois légèrement en-dessous, se trouve l'épine hépatique, dirigée en dehors. Le telson se termine en pointe aiguë et sa longueur égale $2 \frac{1}{3}$ fois sa largeur basale. Il porte à son extrémité libre des soies assez longues, dépassant les épines latéro-internes qui sont 4 fois plus longues que les externes. Les pattes I dépassent en avant l'extrémité de l'écaille d'environ le $1 / 4$ distal du carpe et de toute la longueur de la pince. Cette dernière est contenue $1 \frac{2}{3}$ fois dans la longueur du carpe. Les pattes II sont quelque peu inégales; la grande dépasse le scaphocérite de toute la longueur de sa pince. Les articles sont rugueux, les aspérités, courtes et pointues, de plus, des poils longs et fins y sont disséminés. Voici les dimensions des articles des chélipèdes:

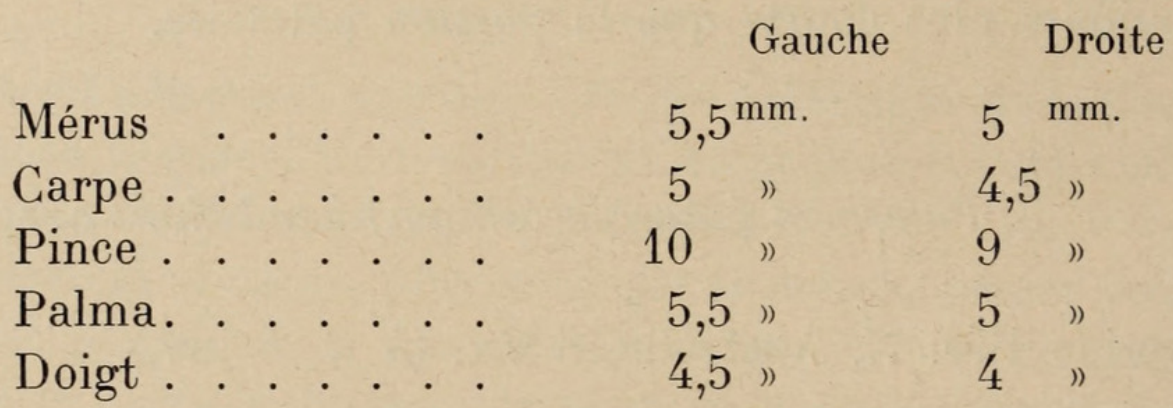

Le mérus, un peu plus long que le carpe, est légèrement renflé en son milieu; sa longueur est contenue approximativement 2 fois dans celle de la pince. Quant au carpe, il s'élargit légèrement en avant. A la pince, la palma est distinctement plus large que l'extrémité distale du carpe; elle est aplatie et sa largeur est d'environ $1 \frac{1}{2}$ fois son épaisseur. Les doigts sont un peu plus courts que la paume et ne sont pas baillants. Au bord interne du doigt mobile $\mathrm{du}$ grand chélipède, on aperçoit au $1 / 3$ inférieur une dent émoussée, précédée de 3-4 petites dentelures basales. Quant au doigt fixe, il porte 1 dent à son $1 / 4$ proximal et cette dent est aussi précédée de 2 petites dents basales. Au petit chélipède, les dents des doigts sont très peu saillantes et les aspérités de leur surface également peu apparentes. Les pattes ambulatoires sont plutôt courtes et garnies de poils fins.

Palaemon handschini vient se placer, comme nous l'avons dit, près de $P$. bariensis de Man et de Pal. callirhoë de Man dont il diffère par la forme et la spinulations du rostre et par la forme de la paume des chélipèdes II qui est moins élargie et moins aplatie. 


\section{Cheraps quadricarinatus (v. Mart.)}

Barrels Kreek, a/ Adelaide Riv., N. Australie, 2.VI.31. 1 ㅇ. Katherine Riv. N. Australie, 1 ô, 1 juv.

Les exemplaires $\hat{\delta}$ et $q$ n'ont que $40^{\mathrm{mm}}$ de longueur et ne sont pas encore adultes. Leur rostre a la forme typique pour l'espèce et présente 2 épines latérales de chaque côté. Le dessus du rostre, légèrement concave, est encore pourvu de nombreux poils fins. Chez le $3^{\text {me }}$ spécimen, plus petit encore que les autres, la partie antérieure du céphalothorax et le rostre sont anormalement développés. Le carène latérale du céphalothorax manque du côté droit et le rostre, élargi à sa base, se divise en avant en deux pointes divergentes. La pointe de gauche porte de chaque côté 2 épines latérales, celle de droite, par contre, n'en possède qu'une de chaque côté.

Les pinces de ces spécimens correspondent par leur forme à la figure qu'a donnée G. Sмгтн ${ }^{1}$ de cette espèce, répandue également en dehors de l'Australie septentrionale, en Nouvelle-Guinée et aux Iles Arou et Misol ${ }^{2}$.

\section{Paratelphusa (Liotelphusa) transversa (v. Mart.)}

Barrels Kreek, a/ Adelaïde Riv., N. Australie, 2.VI.31, 3 ô, 5 우 juv.

Ces individus, quoique jeunes, correspondent assez bien à la description que Mlle M. Rathiun ${ }^{3}$ donne de cette espèce, sous le nom de Potamon (Geotelphusa) transversum (v. Mart.)

Le plus grand spécimen est une $q$ dont le céphalothorax a les dimensions suivantes: largeur maximum, $30 \mathrm{~mm}$, longueur maximum $23,5^{\mathrm{mm}}$; largeur extraorbitaire $19 \mathrm{~mm}$. Le front a $8 \mathrm{~mm}$; il mesure donc à peu près le $1 / 4$ de la largeur maximum de la carapace.

1 Proceed. Zool. Soc. London, 1912, pl. 24.

${ }^{2}$ Roux, J.: Ueber das Vorkommen der Gattung Cheraps auf der Insel Misol. Zool. Anzeiger, Bd. 14, 1914, p. 97.

${ }^{3}$ Rath вun, M. Nouvelles Arch. Museum, Paris, 4 ${ }^{\text {me }}$ série, vol. 7, 1905, p. 215. 
En se repliant vers le bas, il forme une cassure nette; le bord orbitaire qui, vu d'en haut est concave en sa partie médiane forme, vu de face, une ligne transversale. Le bord vrai du front n'est pas visible d'en haut; il est ourlé, comme les côtés latéraux et le bord orbitaire, d'un liseré brunâtre.

Chez le jeune $\widehat{\jmath}$, dont le céphalothorax mesure $25 \mathrm{~mm}$ de largeur, le segment pénultième de l'abdomen est plus large que long et ses bords latéraux sont sub-parallèles. Le segment terminal est un peu plus long que le précédent et sa longueur est égale à sa largeur basale.

Chez quelques exemplaires, le céphalothorax et les pattes sont couverts de macules d'un rouge-brun. Sur le dos de la carapace, ces taches sont plus petites et plus serrées dans les régions antérieures que dans la portion cardiaque. 


\section{$2 \mathrm{BHL}$ Biodiversity Heritage Library}

Roux, J. 1933. "Notes sur quelques Crustacés décapodes d'eau douce provenant de l'Australie septentrionale." Revue suisse de zoologie 40, 343-348. https://doi.org/10.5962/bhl.part.117659.

View This Item Online: https://www.biodiversitylibrary.org/item/148564

DOI: https://doi.org/10.5962/bhl.part.117659

Permalink: https://www.biodiversitylibrary.org/partpdf/117659

\section{Holding Institution}

American Museum of Natural History Library

\section{Sponsored by}

BHL-SIL-FEDLINK

\section{Copyright \& Reuse}

Copyright Status: In copyright. Digitized with the permission of the rights holder.

Rights Holder: Muséum d'histoire naturelle - Ville de Genève

This document was created from content at the Biodiversity Heritage Library, the world's largest open access digital library for biodiversity literature and archives. Visit BHL at https://www.biodiversitylibrary.org. 